\title{
Fractional weighted problems with a general nonlinearity or with concave-convex nonlinearities
}

\author{
Luigi Appolloni ${ }^{1}$ and Dimitri Mugnai ${ }^{2}$ \\ ${ }^{1}$ Università degli Studi di Milano-Bicocca \\ ${ }^{2}$ University of Tuscia Department of Biological and Ecological Sciences
}

September 5, 2020

\begin{abstract}
We consider nonlocal problems in which the leading operator contains a sign-changing weight which can be unbounded. We begin studying the existence and the properties of the first eigenvalue. Then we study a nonlinear problem in which the nonlinearity does not satisfy the usual Ambrosetti-Rabinowitz condition. Finally, we study a problem with general concaveconvex nonlinearities.
\end{abstract}

\section{Hosted file}

Appolloni_Mugnai.pdf available at https://authorea.com/users/356460/articles/479330fractional-weighted-problems-with-a-general-nonlinearity-or-with-concave-convexnonlinearities 